\title{
High Density Lipoprotein (HDL) Cholesterol levels in young pregnant Women
}

\author{
Shishir Kumar Suman', Md Abu Nasar ${ }^{1}$,Rajiv Ranjan Sinha ${ }^{2}$ \\ 1. Assistant Professor, Nalanda Medical College, Patna, India \\ 2. Professor and Head of the Department of Biochemistry, Nalanda Medical College, Patna, India
}

\section{ARTICLE INFO}

\section{Corresponding Author:}

\section{Shishir Kumar Suman \\ Assistant Professor, Nalanda Medical College, Patna, India}

Keywords: Lipoprotein, Pregnancy, HDL Cholesterol, Primipara, Nullipara, LDL Cholesterol.

\begin{abstract}
A woman's reproductive history may affect her risk for coronary heart disease. Parity has been associated with increased coronary disease risk in some studies, while other studies have shown that nulliparous women are at increased risk (1-3). Pregnancy frequency (including frequency of spontaneous abortion $(4,5)$ ) and age at first pregnancy $(6)$ have also been associated with increased coronary disease risk. Although reports of associations of coronary disease risk with parity, age at menarche, or incidence of miscarriage are not all consistent $(7,8)$, the majority of cohort studies have shown an increased risk of coronary disease among women with high gravidity or parity (9). Long-term effects of pregnancy on coronary disease risk factors, such as lipoproteins $(10,11)$, are potential mechanisms for an association between parity and coronary artery disease risk.

Marked increases in lipoprotein concentrations occur during pregnancy (12) and have been correlated with pregnancy-related increases in insulin, 17-beta estradiol, progesterone, and human placental lactogen (13). Total and low density lipoprotein (LDL) cholesterol and triglyceride levels progressively increase during gestation $(10,14)$. Although triglycerides have been reported to decrease rapidly during the postpartum period, total and LDL cholesterol levels may require several months to return to baseline (10, 14). High density lipoprotein (HDL) cholesterol, which has been shown to be inversely associated with coronary disease risk among women $(15,16)$, peaks at mid- gestation and then falls to levels approximately 15 percent above baseline at term (12). Few data are available on the long-term effects of pregnancy on lipoproteins; however, there are reports of inverse associations between parity and postpartum HDL cholesterol levels (17-20). In order to examine further relations of parity with lipid risk factors, we assessed plasma lipids at baseline and at the year 1 and year 2 follow-up examinations among young adult women in an ongoing epidemiologic study.
\end{abstract}

\section{INTRODUCTION \\ MATERIALS AND METHODS \\ Study population}

The Coronary Artery Risk Development in Young Adults (CARDIA) Study is a prospective epidemiologic study designed to identify determinants of the evolution of cardiovascular risk factors among young adults. The study design and characteristics of the cohort have been detailed previously $(21,22)$. In brief, young adults aged $18-30$ years were recruited from different locations in and around Patna, India by community based sampling. Baseline examinations were performed on 5,115 young adults (including 2,787 women), 51 percent of the eligible persons contacted. Recruitment efforts were successful in achieving a study population that was approximately balanced according to age (45 percent aged 18-24 years and 55 percent aged 25-30 years), sex (46 percent men and 54 percent women), race ( 52 percent black and 48 percent white), and education (40 percent having completed $\leq 12$ 
years of education and 60 percent having completed $>12$ years).

Data were available on 91 percent (2,534 women) and 86 percent (2,393 women) of participants from the years 2015 and 2016 follow-up examinations, respectively. In analyses of baseline to year 2 lipid change, we sequentially excluded 402 women, leaving 2,140 available for analyses; some women were excluded for more than one reason. Women who were pregnant at their baseline $(n=5)$ or year $2(n=$ 82) examinations, women with missing ( $\mathrm{n}=27)$ or inconsistent pregnancy data (e.g., women who reported they had previously been pregnant at baseline but reported they had never been pregnant at year $2, n=108$ ), and women with missing lipid data $(n=99)$ were excluded. Because of the progressive lipid changes previously reported with pregnancy $(10,14)$, we also excluded 279 interim pregnancies of $<28$ weeks gestation and eight interim pregnancies of unknown duration. In addition, because previous data have shown an effect of lactation on postpartum lipoproteins $(11,23,24), 75$ women who were breastfeeding at either examination were also excluded. Finally, we excluded the eight women who reported more than one interim pregnancy between baseline and year 2 . Similar exclusions were used for analyses of year 1 to year 2 lipid change. Because 1.5 percent of female CARDIA participants at year 2 reported a history of diabetes mellitus ( 2 nulliparous, 3 primipa- rous, and 23 parous) and 0.4 percent reported gestational diabetes (2 primiparous and 5 parous), we did not exclude women on the basis of these conditions.

\section{Data collection methods}

At baseline, women were asked in a questionnaire if they had ever been pregnant, and, if so, how many pregnancies resulted in live births. At the follow-up examinations, women were asked if they had been pregnant since their previous examination, and, if so, the duration of gestation and the date of delivery. Women who reported having been pregnant were also asked if they were currently breastfeeding.

All participants were asked to fast for 12 hours prior to the examination; participants who did not fast were excluded from analyses of LDL cholesterol and triglycerides. Venous blood samples were collected in ethylene di amine tetra acetic acid (EDTA) at Nalanda Medical College, Patna, India, Department of Biochemistry. The plasma was isolated and stored at $-70^{\circ} \mathrm{C}$ and shipped to the Clinic Laboratory for lipid determinations. Plasma total cholesterol and triglycerides (25), plasma total HDL cholesterol, (available only at baseline and year 2 (26-28)) were determined with standard laboratory methods (29). $\mathrm{HDL}_{2}$ cholesterol was calculated indirectly as the difference of total HDL cholesterol minus $\mathrm{HDL}_{3}$ cholesterol (available at baseline and year 2). LDL cholesterol was calculated using the Friedewald equation (30). The internal coefficient of variation (expressed as percent) obtained by the laboratory in an analysis of pooled samples for total cholesterol, HDL cholesterol, and triglycerides were, respectively, 2.0, 3.5, and 2.2 at baseline, 1.1, 2.4, and 2.3 at year 1, and 1.7, 2.0, and 1.9 at year 2 . The accuracy of routine cholesterol measurement was monitored by performing weekly blind-split sample comparisons with the Abell-Kendall reference method (31).
Body mass index, waist-hip ratio, average daily alcohol intake, physical activity, smoking status, and current oral contraceptive use were collected at each examination and were included as covariates in adjusted analyses. Body weight was measured in light clothing to the nearest $0.5 \mathrm{lb}$ $(0.23 \mathrm{~kg})$ with a calibrated scale; height (without shoes) was measured to the nearest $0.5 \mathrm{~cm}$ using a vertical ruler. Body mass index was computed as weight (kg)/height $(\mathrm{m})^{2}$. Waist circumference was measured in duplicate at the minimum abdominal girth, and hip circumference was measured in duplicate at the maximal protrusion of the hips at the level of the symphysis pubis. Waist-hip ratio was calculated from the average values of these two variables. Physical activity was assessed using the reported frequency of participation in each of 13 activities during the previous year, weighted by the intensity level of each activity, and then summed to give a total physical activity score (33). Race, age, and highest year of education completed were obtained by questionnaire at baseline. Cigarette smoking status at baseline (never, former, or current smoker) and change in smoking status at the follow-up examinations were obtained by self-report (34), as was oral contraceptive use.

\section{Statistical analysis}

In the cross-sectional analysis at baseline, comparison groups were defined as nulliparous, primiparous, or multiparous based on the reported number of previous pregnancies and live births (duration of gestation was unavailable). In the analyses of change in plasma lipids and lipoproteins, comparison groups were defined as follows: nulliparous, women who were nulliparous at baseline and remained nulliparous during follow-up; primiparous, women who were nulliparous at baseline and who had one pregnancy of $\geq 28$ weeks duration between examinations of interest; multiparous, women who were parous at baseline and who had one pregnancy of $\geq 28$ weeks duration between examinations; and parous, women who were parous at baseline and who had no further pregnancies during follow-up. Analyses of change in lipids/lipoproteins were performed comparing year 1 to baseline values and were repeated comparing year 2 to year 1 to examine consistency of findings. There were too few women remaining in their assigned parity groups from baseline to year 2 to examine 2 -year change.

Baseline characteristics (covariates) were compared between groups using Fisher's exact test of proportions and analysis of variance, with Dunnett's multiple comparison tests for pairwise comparisons (using the nulliparous group as the reference) where appropriate (35). To examine differences in lipids/lipoproteins based on parity group, multivariate linear models using Wilk's lambda statistic (36) were used to determine if overall differences existed. To determine parity-related differences in individual lipid or lipoprotein levels, the Roy-Bargmann sequential procedure was used that takes into account the non-independence of univariate $\mathrm{F}$ tests and the inflation of Type 1 error rates. Simultaneous 95 percent confidence intervals were calculated using the Roy union-intersection principal (36) to estimate pairwise differences between parity groups for individual lipid/lipoprotein measures. An overall Type 1 error rate of 0.05 was used and the adjusted Type 1 error for each test in the sequential procedure was 0.01 . Analyses adjusting for race, age, education, body mass index, waist-hip ratio, alcohol consumption, smoking 
status, physical activity, and use of oral contraceptives used similar procedures. There were no significant parity-oral contraceptive use interactions in the analyses.

\section{RESULTS}

Women who were parous at baseline were more often black and current smokers than nullipara (table 1). Primiparous women used oral contraceptives more often than women in the other groups. Women who had more than one child at baseline also had lower physical activity scores, were less well educated, were heavier, and had greater waist-hip ratio than the other groups. There was no parity-related difference in prevalence of alcohol use or in average alcohol intake at baseline. In unadjusted analyses at baseline, HDL cholesterol levels were significantly different between the parity groups (table 2). Multiparous women had significantly lower HDL cholesterol levels than nullipara. The association between parity and HDL cholesterol was diminished but remained statistically significant after adjustment in overall analyses; however, there were no significant pairwise differences between parity groups. A similar finding of overall significance was present for LDL cholesterol in adjusted analyses only. Twoyear change in HDL cholesterol and triglyceride levels was significantly different among the parity groups in overall analyses both before and after adjustment (table 3).
Unadjusted HDL cholesterol decreased in the primipara and this change was significantly different from the increase experienced by parous women who had no further pregnancies during follow-up. There were no significant pairwise differences in HDL cholesterol among parity groups after adjustment and no significant pairwise differences at all for triglycerides. Although not statistically signifi- cant, there was a greater decrease in HDL2 cholesterol fraction in the primipara than in womep in the other parity groups, while the HDL3 cholesterol fraction increased in all groups.

In analyses of the 2-year lipid changes in 1,819 women followed from year 1 to year 2 (table 4), change in HDL cholesterol was again significantly different among the parity groups and remained so after adjustment. The greatest decrease in HDL cholesterol was again present among the primipara compared with both the multiparous and the parous (with no further pregnancies) groups. There were no other significant differences in lipid change between the parity groups. Among 34 primipara who were $\geq 12$ months postpartum at year 2 , the mean \pm standard error unadjusted 2-year decrease in HDL cholesterol $(-7.7$ $\pm 2.5 \mathrm{mg} / \mathrm{dl})$ was greater than that experienced by nullipara $(-0.8 \pm 0.4 \mathrm{mg} / \mathrm{dl}, \mathrm{p}=0.01)$.

TABLE 1. Baseline characteristic of women, based on parity at baseline, CARDIA Study, 2015-2016*

\begin{tabular}{|c|c|c|c|c|}
\hline Characteristic & $\begin{array}{l}\text { Nullparous } \\
(n=1,138]\end{array}$ & $\begin{array}{r}\text { Primiparous } \\
(n=356]\end{array}$ & $\begin{array}{c}\text { MuBlparous } P \\
(n=646]\end{array}$ & value $t$ \\
\hline Black (\%) & 38 & 57 & 68 & $<0.001$ \\
\hline Using oral contraceptives (\%) & 32 & 43 & 28 & $<0.001$ \\
\hline Current smoker (\%) & 21 & 35 & 39 & $<0.001$ \\
\hline Education (years), mean (SD $x^{7}$ ) & $14.4(2.1)$ & $14.0(2.0)$ & $12.9(1.9)$ & $<0.001$ \\
\hline Body mass index $\left(\mathrm{kg} / \mathrm{m}^{2}\right)$, mean (SD) & $23.9(5.2)$ & $24.4(6.0)$ & $25.4(6.2)$ & $<0.001$ \\
\hline Waist-hip ratio, mean (SD) & $0.73(0.05)$ & $0.73(0.05)$ & $0.75(0.05)$ & $<0.001$ \\
\hline Alcohol (ml/day), mean (SD)§. & $7.6(15.9)$ & $6.7(10.9)$ & $6.6(12.9)$ & $<0.34$ \\
\hline Age (years), mean (SD) & $24.1(3.7)$ & $25.1(3.6)$ & $26.2(3.2)$ & $<0.001$ \\
\hline Physical activity score, mean (SD) & $372(262)$ & $337(264)$ & $286(232)$ & $<0.001$ \\
\hline
\end{tabular}

* Nulliparous, women who had never been pregnant at baseline; primiparous, women who had one pregnancy resulting in a live birth prior to baseline; muciparous, women who had two or more pregnancies resulting in a live birth prior to baseline.

$\dagger \quad$ Testing differences between parity group.

$\Varangle \quad$ SD, standard deviation.

$\S \quad$ Includes nondrinkere.

TABLE 2. Baseline mean ( \pm standard error) plasma lipid and lipoprotein levels (mg/dl) of women based on parity at baseline, CARDIA Study, 2015-2016†

\begin{tabular}{|c|c|c|c|c|}
\hline 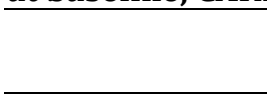 & $\begin{array}{l}\text { NuIlparoua } \\
(n-=1,130)\end{array}$ & $\begin{array}{c}\text { Prim parous } \\
(\mathrm{n}=356)\end{array}$ & $\begin{array}{c}\text { Mutiparoos } \\
(n=646)\end{array}$ & SequenUal $F$ \\
\hline \multicolumn{5}{|l|}{ LDL cholesterol } \\
\hline Crude $x^{\nearrow}$ & $108 \pm 0.9$ & $109 \pm 1.8$ & $109 \pm 1.2$ & 0.27 \\
\hline Adjusted§ & $111 \pm 1.2$ & $109 \pm 1.7$ & $108 \pm 1.4$ & $3.42^{*}$ \\
\hline \multicolumn{5}{|l|}{ HDL cholesterol } \\
\hline Crude $x^{\nearrow}$ & $57 \pm 0.4^{*}$ & $55 \pm 0.7$ & $54 \pm 0.5^{\text {в }}$ & $11.57^{*}$ \\
\hline Adjusted§ & $56 \pm 0.4$ & $55 \pm 0.7$ & $55 \pm 0.5$ & $3.32 *$ \\
\hline \multicolumn{5}{|l|}{$\mathrm{HDL}_{2}$ cholesterol } \\
\hline Crude $x^{\nearrow}$ & $20 \pm 0.3$ & $19 \pm 0.5$ & $18 \pm 0.4$ & 2.00 \\
\hline Adjusted§̧ & $20 \pm 0.3$ & $19 \pm 0.5$ & $18 \pm 0.4$ & 0.50 \\
\hline \multicolumn{5}{|l|}{$\mathrm{HDL}_{3}$ cholesterol } \\
\hline Crude $\nwarrow$ & $36 \pm 0.2$ & $36 \pm 0.3$ & $38 \pm 0.2$ & 1.30 \\
\hline Adjusted§ & $37 \pm 0.2$ & $36 \pm 0.3$ & $37 \pm 0.3$ & 1.10 \\
\hline
\end{tabular}


Triglycerides

\begin{tabular}{lllll} 
Crude $\nearrow$ & $64 \pm 1.0$ & $70 \pm 1.8$ & $70 \pm 1.8$ & 2.25 \\
Adjusted $§$ & $70 \pm 1.2$ & $73 \pm 1.8$ & $72 \pm 1.6$ & 0.88 \\
\hline
\end{tabular}

* $\quad$ Overall $p<0.05$ using the Roy-Bargmann sequential procedure. Significant pairwise

differences

are indicated by differing superscripts.

$† \quad$ Nulliparous, women who had never been pregnant at baseline; primiparous, women who had one pregnancy resulting in a live birth prior to baseline; multiparous, women who had two or more pregnancies resulting in a live birth prior to baseline.

$\chi \quad$ Unadjusted cell means. Hypothesis of no overall effect of baseline parity status on lipid levels using Willis lambda $(\mathrm{p}<0.006)$.

$\S \quad$ Adjusted for oral contraceptive use, race, age, years of education, body mass indeoc, aJcohol consumption, smoking status, physical activity, and waist-hip ratio. Hypothesis of no overall effect of baseline parity status on lipid levels tested using Wilk's lambda $\quad(p=0.016)$.

TABLE 3. Mean ( \pm standard error) 2-year change in plasma lipid and lipoprotein levels (mg/dl) of women baaed on parity at baseline and year 2, CARDIA Study. 2015-16 to 2016-2017†

\begin{tabular}{|c|c|c|c|c|c|}
\hline & $\begin{array}{l}\text { Nullparous } \\
(n=1,083)\end{array}$ & $\begin{array}{l}\text { Primiparous } \\
\text { (nm 67) }\end{array}$ & $\begin{array}{l}\text { Multiparous } \\
(n-137)\end{array}$ & $\begin{array}{l}\text { Parous } \\
(n=865)\end{array}$ & Sequertial $F$ \\
\hline \multicolumn{6}{|c|}{ LDL cholesterol } \\
\hline Crude $x^{\nearrow}$ & $1.2 \pm 0.8$ & $5.9 \pm 2.5$ & $6.4 \pm 2.4$ & $1.6 \pm 0.8$ & 1.72 \\
\hline Adjusted§ & $0.8 \pm 0.9$ & $3.4 \pm 3.1$ & $5.1 \pm 2.2$ & $1.3 \pm 1.0$ & 1.19 \\
\hline \multicolumn{6}{|c|}{ HDL cholesterol } \\
\hline Crude $x^{\nearrow}$ & $2.4 \pm 0.3^{\mathrm{A}}$ & $-3.5 \pm 1.2^{5}$ & $0.4 \pm 0.9$ & $2.5 \pm 0.3^{\mathrm{A}}$ & $9.13^{*}$ \\
\hline Adjusted $\S$ & $2.5 \pm 0.4$ & $-2.6 \pm 1.3$ & $1.0 \pm 0.9$ & $2.8 \pm 0.4$ & $6.77^{*}$ \\
\hline \multicolumn{6}{|c|}{$\mathrm{HDL}_{2}$ cholesterol } \\
\hline Crude $x^{\nearrow}$ & $-0.6 \pm 0.2$ & $-52 \pm 1.0$ & $-2.3 \pm 0.6$ & $-0.3 \pm 0.2$ & 1.88 \\
\hline Adjusted§ & $-0.1 \pm 0.3$ & $-4.1 \pm 1.0$ & $-1.3 \pm 0.7$ & $0.1 \pm 0.3$ & 0.79 \\
\hline \multicolumn{6}{|c|}{$\mathrm{HDL}_{3}$ cholesterol } \\
\hline Crude $x^{\nearrow}$ & $3.0 \pm 0.2$ & $1.7 \pm 0.6$ & $2.6 \pm 0.5$ & $2.6 \pm 0.2$ & 0.21 \\
\hline Adjusted§ & $2.6 \pm 0.2$ & $1.4 \pm 0.7$ & $2.1 \pm 0.5$ & $2.7 \pm 0.2$ & 0.14 \\
\hline \multicolumn{6}{|c|}{ Triglycerides } \\
\hline Crude $x^{\nearrow}$ & $2.9 \pm 0.9$ & $15.5 \pm 3.1$ & $11.6 \pm 3.0$ & $2.6 \pm 1.7$ & $5.52 *$ \\
\hline Adjusted§ & $0.2 \pm 1.5$ & $10.4 \pm 5.1$ & $7.2 \pm 3.5$ & $0.1 \pm 1.6$ & $3.51^{*}$ \\
\hline
\end{tabular}

\begin{tabular}{lllll}
\hline$*$ & Overall $p<0.05$ using the Roy-Bargmann sequential procedure. Significant pairwise & differences & are
\end{tabular} $\dagger \quad$ Nulliparoua, women who had never been pregnant; primiparous, women who were nulliparoua at baseline and who had one pregnancy of $\geq 28$ weeks duration between baseline and year 2 ; multiparous, women who were parous at baseline and who had one pregnancy of $\geq 28$ weeks duration between baseline and year 2; parous, women who were parous at baseline and who had no further pregnancies during follow-up.

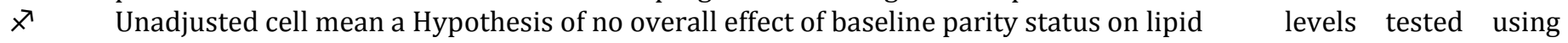
Wait's lambda $(\mathrm{p}<0.001)$.

$\S \quad$ Adjusted for oral contraceptive use, race, age, years of education, body mass index, alcohol consumption, smoking status, physical activity, waist-hip ratio, changes in physical activity, body mass index, and waist-hip ratio. Hypothesis of no overall effect of parity status on lipid levels tested using Wilk's lambda $(p=0.014)$.

TABLE 4. Mean ( \pm standard error) 2-year change In plasma lipid and lipoprotein levels (mg/dl) of women based on parity at baseline, year 1 , and year 2, CARIDA Study, 2015-2016 to 2016-2017†

\begin{tabular}{|c|c|c|c|c|c|}
\hline & $\begin{array}{c}\text { NuBlparous } \\
(n=763)\end{array}$ & $\begin{array}{c}\text { Prtirtparoira } \\
(n-76)\end{array}$ & $\begin{array}{c}\text { MuMparoua } \\
(n=188)\end{array}$ & $\begin{array}{c}\text { Parous } \\
(n=801)\end{array}$ & Sequential $F$ \\
\hline \multicolumn{6}{|c|}{ LDL cholesterol } \\
\hline Crude† & $5.1 \pm 0.9$ & $-4.5 \pm 3.3$ & $-5.8 \pm 2.0$ & $-5.5 \pm 0.9$ & 0.10 \\
\hline Adjusted§ & $5.6 \pm 1.1$ & $-6.8 \pm 2.9$ & $-8.1 \pm 1.9$ & $-5.8 \pm 1.0$ & 0.21 \\
\hline \multicolumn{6}{|c|}{ HDL cholesterol } \\
\hline Crude $\nearrow^{\nearrow}$ & $-0.8 \pm 0.4$ & $-5.2 \pm 1.5^{A}$ & $-2.8 \pm 0.8^{A}$ & $0.5 \pm 0.4^{\mathrm{B}}$ & $10.96 *$ \\
\hline Adjusted§ & $-0.8 \pm 0.4$ & $-4.0 \pm 1.2^{\mathrm{A}}$ & $-2.3 \pm 0.8^{A}$ & $0.8 \pm 0.4^{\mathrm{B}}$ & $8.31^{*}$ \\
\hline \multicolumn{6}{|l|}{ Triglycerides } \\
\hline Crude $x^{\nearrow}$ & $-4.4 \pm 1.2$ & $3.6 \pm 4.2$ & $1.6 \pm 3.1$ & $-2.1 \pm 1.7$ & 1.49 \\
\hline Adjusted§ & $-4.3 \pm 1.7$ & $-0.3 \pm 4.7$ & $3.0 \pm 3.1$ & $-4.0 \pm 1.6$ & 0.62 \\
\hline
\end{tabular}


* $\quad$ Overall $p<0.05$ using the Roy-Bargmann sequential procedure. Significant pairwise indicated by differing superscripts.

$\dagger \quad$ Nulliparoua, women who had never been pregnant; primiparous, women who were nulliparous at year 1 and who had one pregnancy of $\geq 28$ weeks duration between year 1 and year 2 ; multiparous, women who were parous at year 1 and who had one pregnancy of $\geq 28$ weeks duration between year 1 and year 2 ; parous, women who were parous at year 1 and who had no further pregnancies.

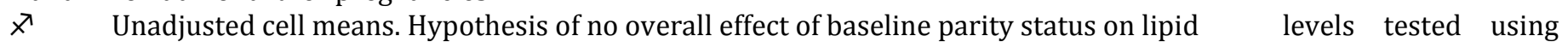
Wilk's lambda $(p<0.001)$.

$\S \quad$ Adjusted ratio for oral contraceptive use, race, age, years ot education, body mass index, alcohol consumption, smoking status, physical activity, waist-hip ratio, changes in physical activity, body mass index, and waist-hip ratio. Hypothesis of no overall effect of parity status on lipid levels tested using Wilk's lambda $(p=0.008)$.

\section{DISCUSSION}

Among healthy reproductive-aged CARDLA participants, pregnancy, and particularly a first pregnancy was associated with adverse changes in HDL cholesterol levels. The decrease in HDL cholesterol appeared to be mainly in the $\mathrm{HDL}_{2}$ fraction. These findings were independent of change in body mass index, waist-hip ratio, and other factors associated with lipoprotein levels. Adverse changes in HDL cholesterol levels in women experiencing a pregnancy during follow-up were of similar magnitude in analyses using pregnancies experienced between the baseline and year 1 and between the year 1 and year 2 examinations. Finally, there were significant inverse associations between parity and HDL cholesterol in crosssectional analyses at baseline; however, the difference was greatest between multipara and nullipara. There were no consistent associations between parity and LDL cholesterol or triglyceride levels.

The duration of the observed effect of pregnancy on HDL cholesterol is unknown. Most women were examined at least 3 months postpartum in CARDIA; however, the effect was probably present for at least one year. In unadjusted analyses of women $\geq 12$ months postpartum at year 2 (year 2-year 1 comparison), the decrease in HDL cholesterol in primipara was significantly greater than that in nullipara and was of similar magnitude to the results presented using all women regardless of time postpartum. Because women frequently changed their parity status during follow-up, we were unable to extend our analyses of year 1-baseline change to the full 2 years of follow-up.

Most previous reports of the effects of pregnancy on Lipoproteins have either not included data from the postpartum period, or have included data from only the first few weeks to months postpartum. However, in longitudinal analyses reported by van Stiphout et al. (17), parous women at one year postpartum had lower HDL cholesterol levels and lower ratios of HDL-to- total cholesterol compared with their prepregnancy levels. Cross-sectional comparisons of never to previously pregnant women indicated that pregnancy was inversely associated with HDL cholesterol levels. In contrast to our findings of no parity-oral contraceptive interaction, the adverse effects on HDL cholesterol were observed mainly among oral contraceptive users in thi;s study. Other longitudinal $(18,20)$ and cross- sectional $(19,37)$ data have shown inverse associations between multiparity and HDL cholesterol. On the other hand, Jimenez et al. (38) have reported that pregnancy did not affect HDL cholesterol levels among 60 women followed through a normal pregnancy and up to 40 days postpartum; however, the follow-up period in this study may not have been long enough to conclude that no effect was present.
Our data indicate that pregnancy, and particularly birth of a first child, may affect women's HDL cholesterol levels, and, possibly through these levels, their risk for cardiovascular disease. The effects of parity on risk for cardiovascular disease have been examined in case-control studies $(3-6,39)$, in case reports $(7,40)$, and in mortality studies (1) with varying results; however, a recent review of the literature concluded that there is an increased risk of coronary disease with a high rtumber of reproductive events (9). The influence of parity on lipoprotein levels and the resultant effects on cardiovascular disease risk have not been thoroughly explored in the context of the available literature. As noted by others $(12,41)$, more data are needed to clarify these issues, particularly with respect to the influence of socioeconomic status on cardiovascular risk and on childbearing practices.

Mechanisms for a putative effect of pregnancy on lipids are speculative. Some have proposed that genetic differences or incipient dyslipidemias may explain "excessive alterations" in lipoprotein levels associated with pregnancy $(14,42)$; however, it is unlikely that the prevalence of these disorders is high enough to explain associations of parity with lipoproteins (specifically with HDL cholesterol) observed in several different study populations.

Another potential mechanism is pregnancy-related metabolic or endocrinc changes that persist in the postpartum period. Reports have indicated that parity is inversely associated with serum de hydro epiandrosterone sulfate (DHEAS) and de hydro epiandrosterone (DHEA) levels, a finding that may only be associated with the first pregnancy and not parity per se $(43,44)$. While few studies have examined whether or not sex steroid hormone levels are predictive of disease in women (45), recent studies in men have shown an inverse association between DHEAS levels and myocardial infarction (46). The associations of DHEA and DHEAS with cardiovascular risk factors in women are not, however, clear (47). The higher androgen (testosterone and DHEAS) levels seen in women with polycystic ovary syndrome have been associated with low mean HDL cholesterol and high mean serum triglyceride and very low density lipoprotein levels (48). On the other hand, the findings on relations between DHEA levels, abdominal obesity, and insulin resistance in women are not consistent $(49,50)$.

Another possible endocrine mechanism is a prolonged effect of pregnancy on insulin resistance or insulin metabolism. It has been observed that insulin resistance develops in the later stages of normal pregnancy when HDL cholesterol levels appear to drop from mid-gestational peaks (13) and that parity may be associated with the later development of diabetes mellitus (51). However, others have reported that parity is not associated with an 
increased risk of subsequent non-insulin-dependent diabetes mellitus (52) and, therefore, may not be associated with insulin resistance in the long term. Thus, although potential long- term effects of pregnancy on lipids could be hormonally mediated, the precise mechanisms are not as yet clear.

Another potential mechanism for an effect of parity on HDL cholesterol is that pregnancy and childbearing could alter body composition or fat distribution. Previous analyses of CARDIA data have shown that primiparas gained 2 to $3 \mathrm{~kg}$ more weight over 5 years of follow-up than either nulliparous or multiparous women (52). Primiparas also had greater increases in waist-hip ratio that were independent of weight gain than the other groups. Although the findings reported here were independent of body mass index and waist- hip ratio, it is still possible that greater adiposity and/or a more central distribution of body fat could mediate the adverse effect of a first pregnancy on HDL cholesterol. More direct measures of body composition and/or fat distribution, such as computed tomography to determine visceral fat, might detect such a relation if it is indeed present. Finally, changes in life-style/behavioral factors due to pregnancy and/or childbearing, such as changes in dietary habits, could also explain our findings. Further studies will be required to explore these potential mechanisms.

Interpretation of our data is limited by several factors. First, the relatively small number of women available for subgroup analysis restricts interpretation. Our power to examine the duration of the effect in the postpartum period was limited; nevertheless, the findings among primipara $\geq 12$ months postpartum at year 5 were consistent with the results overall. We could not examine associations over longer periods (baseline to year 5) due to limited numbers of subjects in some parity groups. Our findings are nevertheless consistent with those reported by others among women followed for 12 months postpartum (17).

Second, the effects of health behaviors were incompletely addressed in our analyses. For example, we were unable to examine the effects of dietary change. In addition, although we did adjust for a number of covariates, we cannot rule out residual confounding due to these factors. Nevertheless, there were significant differences in HDL cholesterol change between the parity groups after adjustment.

The length of time of breastfeeding prior to CARDIA examinations was also not available; therefore, residual effects of lactation could not be determined. Because there were no differences in lipoproteins among parous women breastfeeding compared with nulliparous nonbreastfeeding women at baseline (data not shown), this is not a likely explanation for our findings. Furthermore, in comparison to women who do not breastfeed, lactation has been associated with higher HDL cholesterol concentrations $(11,23)$ and with a more rapid return to baseline of triglycerides (54). Therefore, if present in our data, such an effect of lactation would have led us to underestimate the inverse association between parity and HDL cholesterol. Thus, in spite of these limitations, an adverse effect of a first pregnancy on HDL cholesterol level of potentially significant public health impact was found.

In summary, our results were consistent with reported pregnancy-associated decreases in HDL cholesterol in women who had their first child. Future studies are needed to examine further long-term effects of parity on HDL cholesterol levels, possible mechanisms for these effects, and the potential associations between these changes and coronary disease risk.

\section{ACKNOWLEDGEMENT}

This work was supported by all the faculty staffs and technical staffs of Department of Biochemistry and all other related departments of Nalanda Medical College Hospital, Patna, India.

\section{REFERENCES}

1. Beral V. Long term effects of childbearing on health. J Epidemiol Community Health 1985;39:343-6.

2. Ness RB, Harris T, Cobb J, et aJ. Number of pregnancies and the subsequent risk of Engl J Med 1993;328:1528-33.

3. Talbot EO, Kuller LH, Detre K, et al. Reproductive history of women dying of sudden cardiac death: a casecontrolled study. Int J Epidemiol 1989;18:589-94.

4. Winkelstein W, Stenchever MA, Lilienfeld AM. Occurrence of pregnancy, abortion, and artificial menopause among women with coronary artery disease: a preliminary study. J Chronic Dis 1958;7:273-86.

5. Bengtsson C, Rybo G, Westerberg H. Number of pregnancies, use of oral contraceptives and menopausal age in women with ischemic heart disease, compared to a population sample of women. Acta Med Scand 1973;549:75-81.

6. Beard CM, Fuster V, Annegers JF. Reproductive history in women with coronary heart disease. Am J Epidemiol $1984 ; 120: 108-14$.

7. Oliver MF. Ischemic heart disease in young women. $\mathrm{Br}$ Med J 1974;4:253-9.

8. Colditz GA, Willett WC, Slampfer MJ, et al. A prospective study of age at menarche, parity, age at first birth, and coronary heart disease in women. Am J Epidemiol 1987; 126: 861-70.

9. Ness RB, Schotland HM, Flegal KM, et al. Reproductive history and coronary heart disease risk in women Epidemiol Rev 1994;16:298-314.

10. Potter JA, Nestel PJ. The hyperlipidemia of pregnancy in normal and complicated pregnancies. Am J Obstet Gynecol 1979;133:165-70.

11. Knopp RH, Bergelin RO, Wahl PW, et al. Effects of pregnancy, postpartum lactation, and oral contraceptive use on the lipoprotein cholesterol/triglyceride ratio. Metabolism 1985; 34:893-9.

12. Miller VT. Dyslipoproteinemia in women. Endocrinol Metabol Clin North Am 1990;19:381-8.

13. Desoye G, Schweditsch MO, Pfeiffer KP, et al. Correlation of hormones with lipid and lipoprotein levels 
during normal pregnancy and postpartum. J Gin Endocrinol Metabol 1987; 64:704-12.

14. Knopp RH, Bergelin RO, Wahl PW, et al. Populationbased lipoprotein lipid reference values for pregnant women compared to nonpregnant women classified by sex hormone usage. Am J ～Obstet Gynecol 1982;143:626-37.

15. Bush TL, Fried LP, Barren-Connor E. Cholesterol, lipoproteins, and coronary heart disease in women. Clin Chem 1988; 34(8)B60-B70.

16. Bush TL. Influences on cholesterol and lipoprotein levels in women. Cholesterol and Coronary Disease...Reducing the Risk 1990;2(6):1-6.

17. van Stiphout WAHJ, Hofman A, de Bruijn AM. Serum lipids in young women before, during, and after pregnancy. Am J Epidemiol 1987;126:922-8.

18. Hubert HB, Eaker ED, Garrison RJ, et al. Life-style correlates of risk factors change in young adults: an eightyear study of coronary heart disease risk factors in the Framingham off spring. Am J Epidemiol 1987;125:812-31.

19. Kritz-Silverstein D, Barren-Connor E, Wingard DL. The relationship between multiparity and lipoprotein levels in older women. J Clin Epidemiol 1992;45:761-7.

20. Haertel V, Heiss G, Filipiak B, et al. Cross-sectional and longitudinal associations between high density lipoprotein cholesterol and women's employment. Am J Epidemiol 1992; 135:68-78.

21. Cutter GR, Burke GL, Dyer AR, et al. Cardiovascular risk factors in young adults: the CARD1A baseline monograph. Control Clin Trials 1991; 12(Suppl): 1S-77S.

22. Friedman GD, Cutter GR, Donahue RP, et al. CARDIA: study design, recruitment, and some characteristics of the examined subjects. J Clin Epidemiol 1988;41:1105-16.

23. Knopp RH, Walden CE, Wahl PW, et al. Effect of postpartum lactation on lipoprotein lipids and apoproteins. J Clin Endocrinol Metabol 1985;60:542-7.

24. Fahraeus L, Larsson-Cohn U, Wallentin L. Plasma lipoproteins including high density lipoproteins subtractions during normal pregnancy. Obstet Gynecol 1985;66:468-72.

25. Wamick GR. Enzymatic methods for quantification of lipoprotein lipids. Methods Enzymol 1986;129:10123.

26. Warnick GR, Benderson J, Albcrs JJ. Dextran sulphate$\mathrm{Mg}^{2+}$ precipitation procedure for quantitation of highdensity- lipoprotein cholesterol. Clin Chem 1982;28:137988.

27. Wamick GR, Benderson J, Albers JJ. Quantitation of high density lipoprotein subclasses after separation by dextran sulfate and $\mathrm{Mg}^{2+}$. (Abstract). Clin Chem I982;28:1574.
28. Bachorik PS, Albers JJ. Precipitation methods for quantification of lipoproteins. Methods Enzymol 1986;129:78-100.

29. Donahue RP, Jacobs DR Jr, Sidney S, et al. Distribution of lipoproteins and apolipoproteins by young adults: the CARDIA Study. Atherosclerosis 1989;9:656-64.

30. Friedewald WT, Levy Rl, Fredrickson DS. Estimation of the concentration of low-density lipoprotein cholesterol in plasma, without use of the preparative ultracentrifuge. Clin Chem 1972;18:499-501.

31. Abell LL, Levy BB, Brodie BB, et al. A simplified method for the estimation of total $c$ holesterol in serum and demonstration of its specificity. J Biol Chem 1952;195:35766.

32. Dyer AR, Cutter GR, Liu K, et al. Alcohol intake and blood pressure in young adults: the CARDIA Study. J Clin Epidemiol 1990;43:1-13.

33. Jacobs DR, Hahn LP, Haskell et al. Validity and reliability of short physical activity history: CARDIA and the Minnesota Heart Health Program. J Cardiopulm Rehab 1989;9:448-59.

34. Wagenknecht LE, Perkins LL, Cutter GR, et al. Cigarette smoking behavior is strongly related to educational status: the CARDIA Study. Prev Med 1990;19:158-69.

35. Woolson RD. Comparing more than two groups of observations: analysis of variance for comparing groups. In: Barnett V, Bradley RA, eds. Statistical methods for the analysis of biomedical data. New York: John Wiley \& Sons, 1987:314-63.

36. Morrison DF. Multivariate statistical methods. New York: McGraw-Hill Publishing Co, 1990:254-5.

37. Flegal KM, Ness RB, Kramer RA. Parity and high density lipoprotein (HDL) cholesterol levels in white women from the Second National Health and Nutrition Examination Survey (NHANES II). (Abstract). Am J Epidemiol 1990; 132:766.

38. Jimenez DM, Pocovi M, Ramon-Cajal J, et al. Longitudinal study of plasma lipids and lipoprotein cholesterol in normal pregnancy and puerpcrium. Gynecol Obstet Invest 1988;25: 158-64.

39. Winkelstein W, Rekatc AC. Age trend of mortality from coronary artery disease in women and observations on the reproductive patterns of those affected. Am Heart J 1964;67: 481-8.

40. Engel HJ, Page HL, Campbell B. Coronary artery disease in young women. JAMA 1974;230:1531-4.

41. Barrett-Connor E, Bush TL. Estrogen and coronary heart disease in women. JAMA 1991;265:1861-7.

42. Monies A, Walden CE, Knopp RH, et al. Physiologic and supraphysiologic increases in lipoprotein lipids and apoproteins in late pregnancy and postpartum: possible 
markers for the diagnosis of "prelipemia." Arteriosclerosis 1984;4: 407-17.

43. Key TJA, Pike MC, Wang DY, et al. Long term effects of a first pregnancy on serum concentrations of dehydroepiandro- sterone sulfate and dehydroepiandrosterone. J Clin Endrocri- nol Metab 1990;70:1651-3.

44. Musey VC, Collins DC, Brogan DR, et al. Long term effccts of a first pregnancy on the hormonal environment: estrogens and androgens, i Clin Endocrinol Metab 1987;64:1 11-18.

45. Howard BV, Schneidcrman N, Falkner B, et al. Insulin, health behavior, and lipid metabolism. Metabolism 1993;42:25-35.

46. MitcheLl LE, Sprecher DL, Borecki IB, et al. Evidence for an association between dehydroepiandrosterone sulfate and nonfatal premature myocardial infarction in males.

Circulation 1993;89:89-93.

47. Haffner SM, Valdez RA. Endogenous sex hormones: impact on lipids, lipoproteins, and insulin. Am J Med 1995;98:40S- 47S.

48. Wild RA, Painter PC, Coulson PB, et al. Lipoprotein lipid concentrations and cardiovascular risk in women with polycystic ovary syndrome. J Clin Endocrinol Metab 1985;61: 946-51.

49. Ebeling P, Koivisto VA. Physiological importance of dehydroepiandrosterone. Lancet 1994;343:1479-81.

50. DePergola G, Triggiani V, Giorgino F, et al. The free testosterone to dehydroepiandrosterone sulfate molar ratio as a marker of visceral fat accumulation in premenopausal obese women. Int J Obes 1994;18:65964.

51. Kritz-Silverstein D, Barrett-Connor E, Wingard DL. The effect of parity on the later development of noninsulin-dependent diabetes mellitus or impaired glucose tolerance. N Engl J Med 1989;321:1214-19.

52. Manson JE, Rimm EB, Colditz GA, et al. Parity and incidence of non-insulin-dependent diabetes

mellitus. Am J Med 1992; 93:13-18.

53. Smith DE, Lewis CE, Caveny JL, et al. Longitudinal changes in adiposity associated with pregnancy: the CARDIA study. JAMA 1994;271:1747-51.

54. Darmady JM, Postle AD. Lipid metabolism in pregnancy. Br J Obstet Gynecol 1982;89:211- 15. 
High Density Lipoprotein (HDL) Cholesterol levels in young pregnant Women 